\title{
Extremality conditions for isolated and dynamical horizons
}

\author{
Ivan Booth* \\ Department of Mathematics and Statistics, Memorial University of Newfoundland St. John's, \\ Newfoundland and Labrador, AlC 5S7, Canada \\ Stephen Fairhurst ${ }^{\dagger}$ \\ Department of Physics, University of Wisconsin-Milwaukee Milwaukee, Wisconsin, 53201, USA, \\ and LIGO-California Institute of Technology, Pasadena, California 91125, USA, \\ and School of Physics and Astronomy, Cardiff University, Cardiff, CF2 3YB, United Kingdom
}

(Received 14 September 2007; published 7 April 2008)

\begin{abstract}
A maximally rotating Kerr black hole is said to be extremal. In this paper we introduce the corresponding restrictions for isolated and dynamical horizons. These reduce to the standard notions for Kerr but in general do not require the horizon to be either stationary or rotationally symmetric. We consider physical implications and applications of these results. In particular we introduce a parameter $e$ which characterizes how close a horizon is to extremality and should be calculable in numerical simulations.
\end{abstract}

DOI: 10.1103/PhysRevD.77.084005

PACS numbers: $04.70 . \mathrm{Bw}$

\section{INTRODUCTION}

It is well known that there is a limit on the maximum allowed angular momentum for a Kerr black hole. If such a hole has mass $M$ then the angular momentum $J$ must satisfy $J \leq M^{2}$. Solutions which saturate this bound are known as extremal while Kerr spacetimes with $J>M^{2}$ contain naked singularities rather than black holes. Given this constraint on stationary solutions it is natural to consider whether there is a similar restriction for astrophysical black holes. In contrast to the Kerr holes which sit alone in an otherwise empty universe, real black holes do not exist solely in isolation and can, for example, be surrounded by accretion disks or be components of binary systems.

For this reason, it is interesting to investigate whether extremality conditions can be formulated and applied to interacting black holes. This is of particular interest during black hole collisions. It is widely accepted that following a merger, the final black hole will settle down to one of the known stationary solutions. However, during the highly dynamical merger phase, it is not clear whether the black hole's angular momentum is bounded. The existence or lack of an extremality condition may help us to understand the physics of black hole mergers, and provide insight into whether binary black holes will necessarily "hang up" in orbit, emitting excess angular momentum prior to forming a common horizon. Similar questions arise for black holes forming from the gravitational collapse of matter.

Away from the Kerr-Newmann family of solutions there are some recent results that either support or cast doubt on the possible existence of such a bound. In support, Dain [13] has shown that for a large class of asymptotically flat, axially symmetric, vacuum black holes $J_{\mathrm{ADM}} \leq M_{\mathrm{ADM}}^{2}$

\footnotetext{
*ibooth@math.mun.ca

†Stephen.Fairhurst@astro.cf.ac.uk
}

where the subscripts indicate that these are the ADM mass and angular momentum as measured at spatial infinity. By contrast Petroff and Ansorg [4-6] have recently generated numerical examples of black holes surrounded by rotating rings of matter for which the Komar mass and angular momentum violate the bound $J_{\mathrm{Komar}} \leq M_{\mathrm{Komar}}^{2}$.

Clearly in considering these issues one needs to be careful about how the physical quantities are defined. In particular, in formulating a bound one would like to distinguish between the mass and angular momentum directly associated with the black hole versus any matter or gravitational waves surrounding it. This is, of course, easier said than done. Mass and energy are notoriously ambiguous quantities in general relativity. They are well-defined for entire asymptotically flat spacetimes but in general it is not possible to assign mass and energy to more localized regions of spacetime (see, for example, the discussion in [7]). Similar problems arise for angular momentum and away from axisymmetry it is not at all clear that angular momentum can be described by a single number.

Distinguishing between local and global properties of black holes is one of the main motivations for the recent interest in quasilocal characterizations of horizons, including trapping [8-11], isolated [12-16], and dynamical $[17,18]$ horizons. In this paper we apply the machinery developed in the study of quasilocal horizons to investigate local characterizations of extremality.

We will argue that the ambiguities in defining mass and angular momentum mean that, in general, the usual Kerr extremality bound is not well formulated for quasilocal horizons. Thus we will examine alternative characterizations of extremality and show that they do apply. For isolated horizons these arise from (1) the non-negativity of the surface gravity and (2) the idea that there should be trapped surfaces just inside the horizon. For dynamical horizons only the second these characterizations is appli- 
cable since the surface gravity is only meaningful for slowly evolving dynamical horizons (in the sense of [1921]).

Both the surface gravity and trapped surface characterizations of extremality give rise to an alternative, local extremality condition. This condition is similar in spirit to the Kerr extremality bound, and involves contributions from both the black hole's angular momentum and horizon matter fields. However, it can be written locally on the horizon and angular momentum ambiguities are avoided by making use of the square of an "angular momentum density" integrated over the horizon rather than the angular momentum relative to any particular axis. With this definition of extremality, we show that generic dynamical horizons, in the sense of [22], are necessarily subextremal. Isolated horizons can obtain extremality (in which case the local geometry must be that of the Kerr horizon [23]). We discuss how this local extremality compares to the standard Kerr relation, and argue that in those cases where both are well formulated it may still be possible for a black hole to violate the Kerr bound.

The paper is laid out as follows. We begin in Sec. II with a discussion of extremality for stationary black holes and recall the three notions of extremality already mentioned: maximum angular momentum, vanishing surface gravity, and coincidence of the inner and outer horizons. The next section shows how these notions may be adapted to isolated horizons, examines conditions under which they are equivalent, and considers situations under which one or more of them might be violated. In Sec. IV we use this experience to study the equivalent notions for dynamical horizons and show that these horizons are always subextremal. Finally, Sec. V provides a brief summary. An appendix shows how the various notions apply to the Kerr (anti-)deSitter family of solutions.

\section{STATIONARY BLACK HOLE HORIZONS}

Let us begin by reviewing the notion of extremality for stationary, asymptotically flat black hole spacetimes. In Einstein-Maxwell theory, the uniqueness theorems tell us that the class of such solutions is restricted to the KerrNewman spacetimes. Furthermore, these spacetimes are characterized by only three quantities; their mass $\mathrm{M}$, angular momentum $\mathrm{J}$ and electric charge Q. Black hole solutions exist for all values of $M, J$ and $Q$ which satisfy the inequality:

$$
a^{2}+Q^{2} \leq M^{2} \quad \text { where } a=J / M .
$$

If this inequality is violated the resulting spacetimes are still solutions of the Einstein equations, however they contain a naked singularity in lieu of a black hole. The first notion of extremality arises from Eq. (1). Solutions for which the equality is satisfied, namely

$$
a^{2}+Q^{2}=M^{2}
$$

are said to be extremal as they contain the maximum allowed angular momentum/charge for a given mass.

Since the Kerr-Newman solutions are stationary and axisymmetric, they have both a time-translation Killing vector field $t^{a}$ and a rotational Killing vector field $\phi^{a}$. The event horizon is a nonexpanding null surface whose null normal is

$$
\xi^{a}=t^{a}+\Omega \phi^{a},
$$

where $\Omega$ is interpreted as the angular velocity of the horizon. It is then straightforward to calculate the acceleration of $\xi$ at the horizon:

$$
\xi^{b} \nabla_{b} \xi^{a}=\kappa \xi^{a} .
$$

The quantity $\kappa$ is known as the surface gravity. Since $\xi$ is defined in terms of Killing vectors which are appropriately normalized at infinity, there is no ambiguity in its normalization and by direct calculation

$$
\kappa=\frac{\left(M^{2}-a^{2}-Q^{2}\right)^{1 / 2}}{2 M\left[M+\left(M^{2}-a^{2}-Q^{2}\right)^{1 / 2}\right]-Q^{2}} .
$$

The second notion of extremality comes from this surface gravity. It is clear from Eqs. (1) and (5) that the surface gravity is only well-defined for black hole (as opposed to naked singularity) solutions and is necessarily nonnegative. Furthermore, for an extremal black hole satisfying (2), the surface gravity vanishes, i.e. $\kappa=0$. Thus, vanishing surface gravity is often taken as the defining property of an extremal horizon.

Finally, we can understand extremality from the geometric structure of spacetime - one of the fundamental properties of a black hole is that it contains trapped surfaces which are defined in the following way. Any spacelike two-surface has two future-pointing null normals, which we will denote $\ell$ and $n$. Then, the expansion of these null vectors is defined as:

$$
\theta_{(\ell)}=\tilde{q}^{a b} \nabla_{a} \ell_{b} \quad \text { and } \quad \theta_{(n)}=\tilde{q}^{a b} \nabla_{a} n_{b},
$$

where $\tilde{q}_{a b}$ is the metric of the two-surface. On a trapped surface, the expansions of both null vector fields are negative. This is in contrast to a typical (convex) two-surface in flat space which will have one positive and one negative expansion. For asymptotically flat spacetimes, the existence of a trapped surface is sufficient to imply the existence both an event horizon enclosing the surface and a spacetime singularity somewhere in its interior [24].

For typical charged or rotating black holes, there are two horizons, the event horizon and the inner Cauchy horizon. These are null, foliated by two-dimensional marginally trapped surfaces $\left(\theta_{(\ell)}=0\right.$ and $\left.\theta_{(n)}<0\right)$, and split the spacetime into distinct regions. It is only in the region between the horizons that trapped surfaces exist. If the charge or angular momentum is increased towards the extremal value, the trapped region between the horizons shrinks, until, at extremality, the inner and outer horizons 
coincide, the trapped region vanishes and only the marginally trapped surfaces of the horizon remain. It is this notion of extremality which is used in Israel's proof that a nonextremal black hole cannot achieve extremality in a finite time [25].

Thus, we see that for event horizons in stationary spacetimes, there are three notions of horizon extremality which all coincide:

First characterization: The angular momentum and charge of a black hole are restricted according to Eq. (1). For an extremal black hole, $a^{2}+Q^{2}=M^{2}$.

Second characterization: The surface gravity $\kappa$ of a black hole must be greater than or equal to zero. The surface gravity vanishes if and only if the horizon is extremal.

Third characterization: The horizon of the black hole is a marginally trapped surface. For nonextremal black holes, the interior of the black hole must contain trapped surfaces, while for extremal black holes, the inner and outer horizons coincide and there are no trapped surfaces.

In the remainder of this paper, we will argue that the second and third definitions can be extended to isolated and dynamical horizons. Furthermore, we will obtain a horizon relation similar in spirit, though not identical, to the one appearing in the first definition above. We start with isolated horizons.

\section{ISOLATED HORIZONS}

Isolated horizons have been introduced to capture the local physics of the horizon of a black hole in equilibrium [12-16]. These are null surfaces and so form causal boundaries. However, unlike event horizons, they are defined (quasi-)locally. Specifically, a null surface $\Delta$ of topology $S^{2} \times \mathbb{R}$ with (degenerate) metric $q_{a b}$, derivative $D_{a}$, and normal $\ell_{a}$ is an isolated horizon if:

(1) $\Delta$ is nonexpanding: $\theta_{(\ell)}=0$,

(2) an energy condition holds at the horizon: $-T^{a}{ }_{b} \ell^{b}$ is future-directed and causal, and

(3) the null vector $\ell^{a}$ is scaled such that

$$
\left[\mathcal{L}_{\ell}, D\right]=0 .
$$

The energy condition is weaker than and implied by any of the standard energy conditions. Together with the first condition and the Raychaudhuri equation it follows both that the intrinsic geometry of $\Delta$ is invariant in time: $\mathcal{L}_{\ell} q_{a b}=0$ and that there is no flux of matter through the horizon: $T_{a b} \ell^{a} \ell^{b}=0$. The third condition fixes the scaling of $\ell^{a}$ up to an overall constant and ensures that the extrinsic geometry is similarly invariant in time.

To make all of this a little more concrete, note that one can always find functions $v$ on $\Delta$ that are compatible with $\ell$ (so that $\mathcal{L}_{\ell} v=1$ ) and which have spacelike level surfaces $S_{v}$ with topology $S^{2}$. For such a function, $n_{a}=-D_{a} v$ is not only normal to these surfaces of constant $v$ but is also null and satisfies $\ell \cdot n=-1$. The spacelike metric on the $S_{v}$ can be written as $\tilde{q}_{a b}=g_{a b}+\ell_{a} n_{b}+n_{a} \ell_{b}$.

With these additional structures the invariance of the intrinsic geometry can be written as

$$
\mathcal{L}_{\ell} \tilde{q}_{a b}=\frac{1}{2} \theta_{(\ell)} \tilde{q}_{a b}+\sigma_{a b}^{(\ell)}=0,
$$

so that both the expansion $\theta_{(\ell)}$ and shear $\sigma_{a b}^{(\ell)}$ of the twosurfaces vanish. Further, the third condition implies that the corresponding expansion $\theta_{(n)}$ and shear $\sigma_{a b}^{(n)}$ in the $n$-direction are also invariant in time

$$
\mathcal{L}_{\ell} \theta_{(n)}=0 \quad \text { and } \quad \mathcal{L}_{\ell} \sigma_{a b}^{(n)}=0,
$$

as is the connection

$$
\tilde{\omega}_{a}=-\tilde{q}_{a}^{b} n_{c} \nabla_{b} \ell^{c}
$$

on the normal bundle to the foliation two-surfaces:

$$
\mathcal{L}_{\ell} \tilde{\omega}_{a}=0 .
$$

Finally, one can use the axioms to prove a zeroth law. For the allowed scalings of the null vectors, the surface gravity $\kappa$, defined in a similar manner to that on the event horizon (4),

$$
\ell^{b} \nabla_{b} \ell^{a} \equiv \kappa \ell^{a}
$$

is constant on the horizon: $D_{a} \kappa=0$ [14].

On an isolated horizon, the scaling of the null vectors is only fixed up to an overall positive multiplicative constant. Under allowed rescalings $\ell \rightarrow c \ell$ and $n \rightarrow n / c$, the connection $\tilde{\omega}_{a}$ is invariant while $\kappa \rightarrow c \kappa$. Thus, while $\kappa$ is constant over $\Delta$, its exact value is only fixed up to sign (ie. positive, negative, or zero).

Derivations of these facts can be found in the already cited references or in [26] which focuses on the geometry of horizons.

\section{A. Extremality from $Q, a$, and $M$ ?}

We begin with the first notion of extremality: the horizon of a Kerr-Newman black hole is extremal if and only if $a^{2}+Q^{2}=M^{2}$. Let us attempt to extend this to isolated horizons. The prerequisite to this is to obtain a satisfactory definition of each of these quantities and, except for the electric charge, that is where problems arise. Given a twodimensional cross section $S_{v}$ of the horizon, the charge is well-defined by Gauss' law:

$$
Q \equiv \frac{1}{4 \pi} \int_{S_{v}} d^{2} x \sqrt{\tilde{q}} F_{a b} \ell^{a} n^{b}=\frac{1}{4 \pi} \int_{S_{v}} d^{2} x \sqrt{\tilde{q}} E_{\perp},
$$

where $\tilde{q}$ is the determinant of the two-metric $\tilde{q}_{a b}$ on $S_{v}$ and $F_{a b}$ is the electromagnetic field tensor. Equivalently, we rewrite $F_{a b} \ell^{a} n^{b}=F_{a b} \hat{u}^{a} \hat{s}^{b}=: E_{\perp}$ where $\hat{u}$ and $\hat{s}$ respectively are orthogonal timelike and spacelike unit normal vectors to $S_{v}$. $E_{\perp}$ is the flux of the electric field through $S_{v}$ as observed by a timelike observer with evolution vector 
$\hat{u}^{a}$. Since the horizon is isolated, this is independent of the cross section [14].

Next, we consider angular momentum. In classical, nonrelativistic, physics angular momentum is defined relative to an axis of rotation. For isolated horizons the analogue of an axis of rotation is a rotational vector field. Following $[19,21,27]$ this is given by $\phi^{a} \in T S_{v}$ whose flow foliates the $S_{v}$ into closed integral curves of parameter length $2 \pi$ plus two fixed points (the poles of the rotation). A vector field of this type is necessarily divergence-free and the canonical example is a horizon with a rotational Killing vector field $\phi^{a}$ so that

$$
\mathcal{L}_{\phi} \tilde{q}_{a b}=0 .
$$

The angular momentum relative to a rotational vector field $\phi^{a}$ is then $[15,16]$

$$
J[\phi]=\frac{1}{8 \pi G} \int_{S} d^{2} x \sqrt{\tilde{q}} \phi^{a} \tilde{\omega}_{a}+\frac{1}{4 \pi G} \int_{S} d^{2} x \sqrt{\tilde{q}} \phi^{a} A_{a} E_{\perp},
$$

where $\tilde{\omega}_{a}$ is the connection of the normal bundle that we have already encountered in Eq. (10), and $A_{a}$ is the electromagnetic connection. It is immediate that on an isolated horizon this quantity is independent of the choice of cross section $S_{v}$.

This is closely related to other standard measures of angular momentum such as the Brown-York [28] or dynamical horizon [18] measures. In particular, as is discussed in more detail in [14]

$$
D_{a} \ell^{b}=\left(-\kappa n_{a}+\tilde{\omega}_{a}\right) \ell^{b}
$$

is the Weingarten map and is analogous to the standard extrinsic curvature, although tailored to the null surface of the horizon. Then, it is not surprising that the geometric part of the angular momentum (15) agrees with usual extrinsic curvature formula. To see this, consider the case where the isolated horizon is an apparent horizon found in a numerical simulation. In this case, the $S_{v}$ are each contained in spacelike three-surfaces $\Sigma_{t}, \hat{u}^{a}$ is the future directed unit normal to the $\Sigma_{t}$ and $\hat{s}^{a} \in T \Sigma_{t}$ is the outward-pointing spacelike unit normal to the $S_{v}$. Then, for a divergence-free rotational vector field $\phi^{a}$ it is straightforward that the isolated horizon angular momentum can be rewritten as

$$
J[\phi]=\frac{1}{8 \pi G} \int_{S_{v}} d^{2} x \sqrt{\tilde{q}}\left(\phi^{a} \hat{s}^{b} K_{a b}+2 \phi^{a} A_{a} E_{\perp}\right),
$$

where $K_{a b}=h_{a}^{c} h_{b}^{d} \nabla_{c} \hat{u}_{d}$ is the extrinsic curvature of $\Sigma_{t}$ (with $h_{a b}$ the induced three-metric on $\Sigma_{t}$ ). In stationary black hole spacetimes, this measure also agrees with the Komar and ADM angular momenta evaluated at infinity.

Thus, given a rotational vector field $\phi^{a}$ the angular momentum of the horizon is well defined. Unfortunately, in the absence of axisymmetry there is no obvious way to uniquely select a geometrically preferred rotational vector field. Indeed, for highly distorted horizons, it is by no means clear that one should always expect such an "axis of rotation" to exist. For nonaxisymmetric horizons it seems unlikely that the angular momentum can be characterized by a single number (though see [29-33] for alternative viewpoints).

Even if we restrict our attention to axially symmetric horizons, in order to define extremality by Eq. (1) we would still need a definition of horizon mass $M$ and here the greatest difficulties arise. While local definitions of angular momentum for a surface are readily available and tend to agree, the issue of a local energy or mass is much more difficult $[7,14,27]$. In particular, the rescaling freedom of $\ell$ precludes the identification of a preferred energy associated with evolution along $\ell$. A common solution to this is to simply define the mass of a horizon with area $A$ and angular momentum $J$ to be equal to the value it would take in the Kerr spacetime. Then by the Christodoulou formula [34]

$$
M^{2}:=\frac{\left(R_{H}^{2}+Q^{2}\right)^{2}+4 J^{2}}{4 R_{H}^{2}}
$$

where $R_{H}=\sqrt{a /(4 \pi)}$ is the areal radius of the horizon. With this mass, it is straightforward to show that $|J|$ is less than $M^{2}$. However this is simply a property of the definition and the physical relevance of (18) for highly distorted black holes is, at best, unclear.

Given the difficulties with the definition of mass, it probably makes more sense to rephrase any characterization of type (1) entirely in terms of quantities such as $R_{H}$, $Q$, and $J$ which can be locally measured on the horizon. Then, this bound may be rewritten as

$$
Q^{4}+4 J^{2} \leq R_{H}^{4}
$$

Thus defining $Q, J$, and $R_{H}$ as we have above, this is the first possible characterization of extremality, at least for axisymmetric horizons. There is no general derivation that this bound must hold outside of the Kerr-Newman family. Indeed, the inequality in Eq. (19) can be violated for nonasymptotically flat spacetimes (Appendix A). Similarly, in higher dimensional asymptotically flat spacetimes the original bound (1) can also be violated [35,36]. Despite this, Ansorg and Pfister have demonstrated that (19) holds (with equality) for a class of extremal configurations of black holes surrounded by matter rings. Furthermore, they have conjectured that the inequality will hold for stationary, axially and equatorially symmetric black hole-matter ring solutions [37].

\section{B. Extremality from $\kappa$}

The second notion of extremality for Kerr black holes says that the surface gravity is positive for subextremal holes and zero for extremal holes. It is never negative. We now consider this characterization for isolated horizons 
and see that in many ways it is more satisfactory than that considered in the previous section.

The surface gravity for an isolated horizon was given in Eq. (12), where it was noted that a zeroth law holds so that $\kappa=\kappa_{o}$ everywhere on $\Delta$ for some (fixed) $\kappa_{o} \in \mathbb{R}$. The scaling of the null vectors is only fixed up to a positive multiplicative constant so rescalings of the form $\ell \rightarrow c \ell$, $c \in \mathbb{R}^{+}$are allowed. For such rescalings $\kappa \rightarrow c \kappa$ and so the formalism only allows us to say whether an isolated horizon has a surface gravity that is positive, negative, or zero. This is sufficient for our purposes; an isolated horizon is subextremal if and only if $\kappa>0$, extremal if $\kappa=0$, and superextremal if $\kappa<0$.

Such a definition is more than just nomenclature. For $\kappa=0$ there is a local uniqueness theorem for isolated horizons; the intrinsic geometry of an extremal isolated horizon must be identical to that of the Kerr-Newmann horizon with the same area, charge and angular momentum [23]. Further, subextremal horizons must obey bounds on their electric charge and the angular momentum one-form $\tilde{\omega}_{a}$. To see this, note that the evolution of $\theta_{(n)}$ is given by [14,21]:

$$
\begin{aligned}
\mathcal{L}_{\ell} \theta_{(n)}+\kappa \theta_{(n)}+\tilde{R} / 2= & \tilde{d}^{a} \tilde{\omega}_{a}+\tilde{\omega}^{a} \tilde{\omega}_{a} \\
& +(8 \pi G) T_{a b} \ell^{a} n^{b},
\end{aligned}
$$

where $\tilde{R}$ is the two-curvature of the cross sections of the horizon, $\tilde{d}$ is the spacelike two-metric compatible covariant derivative and $T_{a b}$ is the energy-momentum tensor.

The standard definition of an isolated horizon does not restrict the sign of the inward expansion $\theta_{(n)}$. However, since we are interested only in black hole horizons ${ }^{1}$, it is reasonable to impose the extra requirement that there be trapped surfaces "just inside" the horizon. Thus, by continuity we restrict our attention to horizons with $\theta_{(n)}<0$.

Now, consider Eq. (20) evaluated on a subextremal isolated horizon. On the left-hand side, the first term will vanish since the geometry is time independent. The second term is necessarily nonpositive: by assumption surface gravity is non-negative, and we have restricted to black hole horizons where $\theta_{(n)}<0$. Finally, although the $\tilde{R}$ itself can vary in sign, we know that since the cross sections of the horizon have topology $S^{2}, \int_{S_{v}} \sqrt{\tilde{q}} \tilde{R}=8 \pi$.

\footnotetext{
${ }^{1}$ The defining conditions for isolated horizons are intended to be necessary conditions that a null surface should meet in order to be considered as the boundary of a noninteracting black hole region. However, they are not sufficient to distinguish black hole horizons from white hole or cosmological horizons. Furthermore, there are examples of isolated horizons which either do not correspond to black holes (in [38] there are no trapped surfaces) or their black hole status is unclear (in [39] it is not known whether the "black holes" all contain trapped surfaces).
}

Thus, integrating (20) over any $S_{v}$ gives

$$
e:=\frac{1}{4 \pi} \int_{S_{v}} d^{2} x \sqrt{\tilde{q}}\left(8 \pi G T_{a b} \ell^{a} n^{b}+\|\tilde{\omega}\|^{2}\right) \leq 1,
$$

(the integral of the exact derivative $\tilde{d}_{a} \tilde{\omega}^{a}$ vanishes). This gives an alternative characterization of extremality for isolated horizons: $\kappa$ vanishes if and only if $e=1$ and is positive if and only if $e<1$. This expression provides a local extremality condition for isolated horizons expressed in terms of the horizon angular momentum (encoded in the one-form $\tilde{\omega}$ ) and matter fields. As such, it is similar in spirit to the standard Kerr bound. However, this condition is applicable to all isolated horizons, and does not require either axisymmetry or asymptotic flatness.

The exact interpretation of the matter term depends on the matter present at the horizon, but for electromagnetism it is related to the electric and magnetic charge of the hole:

$$
8 \pi G T_{a b} \ell^{a} n^{b}=\left(E_{\perp}^{2}+B_{\perp}^{2}\right) .
$$

The first term is the square of the electric flux density, while the second is the square of the corresponding magnetic flux - the integral of $B_{\perp}$ is the magnetic charge contained by $S_{v}$.

The second term of (21) is associated with angular momentum. It sidesteps the problems of defining an axis of rotation by working with the square of the angular momentum density integrated over the horizon as a measure of the total angular momentum. To gain some insight into this quantity, consider an axisymmetric horizon. On such a horizon, the angular momentum can be decomposed into its multipole moments [40]. Based on this decomposition, $J$ can be interpreted as the dipole angular momentum of the horizon. Then the quantity appearing in our extremality condition (21) can, at least intuitively, be thought of as the sum of the squares of all the angular momentum multipoles of the horizon.

These associations can be made concrete by calculating $e$ for the known black hole solutions. First for ReissnerNordström spacetimes (where $\tilde{\omega}_{a}$ vanishes) it is straightforward to show that

$$
e=Q^{2} / R_{H}^{2}
$$

where $Q$ is the charge and $R_{H}$ is the areal radius of the horizon. Thus $e=1$ corresponds to the extremality condition $Q=R_{H}(=M)$ while $e<1$ implies that $Q<R_{H}$.

Moving on to the Kerr-Newman solutions the functional form of $e$ becomes considerably more complicated and so instead of stating it explicitly, we plot it in Fig. 1 as a function of the angular momentum and electric charge. Recall from Eq. (19) that the standard extremality condition for Kerr-Newman solutions can be written as $Q^{4}+$ $4 J^{2}=R_{H}^{4}$. Then from the figure, we see that for these extremal solutions $e=1$ while for nonextremal solutions $e<1$. Therefore, the local extremality quantity behaves as expected for stationary, asymptotically flat black holes. 


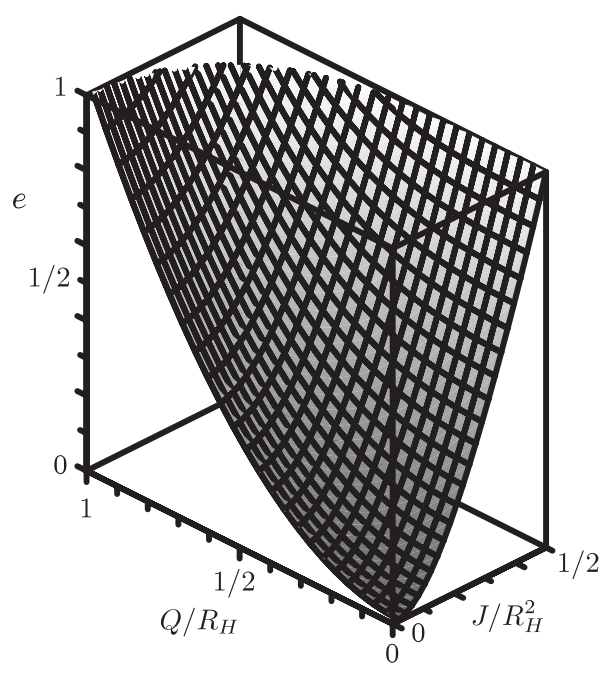

FIG. 1. Plot of the extremality parameter, $e$, for a KerrNewman horizon as a function of the scaled angular momentum $J / R_{H}^{2}$ and electric charge $Q / R_{H}$. For nonspinning black holes, $J=0$ and $e=Q^{2} / R_{H}^{2}$. The extremal Kerr-Newman solutions which satisfy $\left(Q / R_{H}\right)^{4}+4\left(J / R_{H}^{2}\right)^{2}=1$ all have an extremality parameter $e=1$.

\section{Trapped surfaces}

Finally we consider the third characterization of extremality: a horizon is subextremal if there are trapped surfaces "just inside" the horizon and extremal (or superextremal) if there are no such surfaces. For this notion we need to understand how the properties of a $\theta_{(\ell)}=0$ surface change under infinitesimal deformations. Continuing to assume that $\theta_{(n)}<0$ on the horizon (and so by continuity remains negative for sufficiently small deformations), we are then interested in cases where there exists an inward deformation so that $\theta_{(\ell)}$ also becomes negative. The equations governing such deformations have been derived and rederived many times and in many ways over the years [8,21,41-45]. Here we follow [21].

There it is shown that given a spacelike two-surface $S$ and a transverse deformation vector field $X^{a}$,

$$
\delta_{X} \theta_{(\ell)}=-\tilde{d}^{2} B+2 \tilde{\omega}^{a} \tilde{d}_{a} B-B \delta_{n} \theta_{(\ell)}+A \delta_{\ell} \theta_{(\ell)},
$$

where $A$ and $B$ are the components of deformation vector relative to the null normals so that $X^{a}=A \ell^{a}-B n^{a}$. Furthermore,

$$
\delta_{\ell} \theta_{(\ell)}=-\left\|\sigma^{(\ell)}\right\|^{2}-(8 \pi G) T_{a b} \ell^{a} \ell^{b}
$$

(the Raychaudhuri equation with $\theta_{(\ell)}=0$ ) and

$$
\delta_{n} \theta_{(\ell)}=-\tilde{R} / 2+\|\tilde{\omega}\|^{2}-\tilde{d}_{a} \tilde{\omega}^{a}+(8 \pi G) T_{a b} \ell^{a} n^{b},
$$

are the variations of $\theta_{(\ell)}$ under the deformations generated by the null vectors. One way of calculating these deformations is to construct a coordinate system on the manifold for which $S$ is parametrized by two-coordinates [say $(\theta, \phi)]$ and is a level surface with respect to the other two. The deforming vector (be it $X, \ell$, or $n$ ) should be a tangent vector field to one of these other coordinates. For such a construction, quantities such as $\tilde{\omega}_{a}$ can be defined on level surfaces of the non- $(\theta, \phi)$ coordinates in a neighborhood of $S$. Then the $\delta$ s are Lie derivatives. In fact we have already seen an example of this type of construction in this paper; Eq. (20) could equally well be written as $\delta_{\ell} \theta_{(n)}$. Indeed, Eq. (26) can be obtained from (20) by simply switching $\ell$ and $n$ and noting that $\theta_{(\ell)}=0$.

Inward deformation vector fields will necessarily take the form $r=A \ell+B n$ where $B>0$. For simplicity, we can rescale the null vectors such that $B=1$. Let us denote these rescaled vectors as $\bar{\ell}, \bar{n}$. Then

$$
\delta_{r} \theta_{(\ell)}=\delta_{\bar{n}} \theta_{(\bar{\ell})},
$$

since on an isolated horizon $\delta_{\bar{\ell}} \theta_{(\bar{\ell})}=0$ regardless of the scaling on the null vectors [note that this result is independent of Eq. (7)]. Thus, slices of the horizon may be deformed inwards into fully trapped surfaces if and only if there is a scaling of the null vectors such that $\delta_{\bar{n}} \theta_{(\bar{\ell})}<0$.

By this measure we characterize an isolated horizon as subextremal if there exists a scaling of the null vectors such that $\delta_{\bar{n}} \theta_{(\bar{\ell})}<0$, extremal if there exists a scaling such that $\delta_{\bar{n}} \theta_{(\bar{\ell})}=0$, and superextremal if there exists a scaling such that $\delta_{\bar{n}} \theta_{(\bar{\ell})}>0$. It is important to keep in mind that each of these conditions must hold everywhere on $\Delta$ and that it will usually be nontrivial to find the correct scaling needed for the classification.

The Kerr solutions themselves provide an example of these difficulties. For rapidly rotating Kerr black holes with the usual (Killing vector) scaling of the null vectors, $\delta_{n} \theta_{(\ell)}$ varies in sign over $S_{v}$. However, this does not indicate that these horizons lie outside the classification system. Instead it suggests that a different scaling of the null vectors is needed. Such a rescaling is considered explicitly in Appendix C of [21], however here we generalize that calculation to prove a more general result: for axisymmetric isolated horizons with $\theta_{(n)}<0$ the surface gravity and trapped surface classifications of extremality are equivalent. That is

$$
\begin{aligned}
\text { Subextremal: } \kappa>0 \Leftrightarrow e<1 \Leftrightarrow \delta_{\bar{n}} \theta_{(\bar{\ell})}<0, \\
\text { Extremal: } \kappa=0 \Leftrightarrow e=1 \Leftrightarrow \delta_{\bar{n}} \theta_{(\bar{\ell})}=0 \quad \text { and }
\end{aligned}
$$

Superextremal: $\kappa<0 \Leftrightarrow e>1 \Leftrightarrow \delta_{\bar{n}} \theta_{(\bar{\ell})}>0$,

where $\bar{\ell}^{a}$ and $\bar{n}^{a}$ are appropriate rescalings of the null vectors.

We prove this result by explicitly constructing these rescalings. To this end we first note the following key fact: On a topologically spherical two-surface embedded in spacetime there is always a scaling of the null vectors so that the angular momentum one-form is divergence-free: 
$\bar{d}_{a} \tilde{\omega}^{a}=0$ (this result ultimately follows from the Hodge decomposition theorem [26]). The scaling is unique up to the usual multiplicative constant. Thus, taking such a pair of vectors $\left(\ell_{o}^{a}, n_{o}^{a}\right)$ as a reference, we can write any other scaling of the null vectors as

$$
\ell^{a}=f \ell_{o}^{a} \quad \text { and } \quad n^{a}=\frac{1}{f} n_{o}^{a},
$$

for some scalar function $f$ over $S_{v}$. Then we can define the inverse scaling

$$
\bar{\ell}^{a}=\frac{1}{f} \ell_{o}^{a} \quad \text { and } \quad \bar{n}^{a}=f n_{o}^{a}
$$

and using Eqs. (20) and (26) it is straightforward to see that on an isolated horizon:

$$
\delta_{\bar{n}} \theta_{(\bar{\ell})}=\kappa \theta_{(n)}-4 \tilde{\omega}_{o}^{a} \bar{d}_{a} \ln f .
$$

For an axisymmetric horizon the dual requirements that $\tilde{\omega}_{o}^{a}$ respect the symmetry and that $\left\|\tilde{\omega}_{o}\right\|$ not diverge at the poles of rotation imply that $\tilde{\omega}_{o}^{a}$ must be parallel to the rotation vector. Then the last term of (30) vanishes and we find that

$$
\delta_{\bar{n}} \theta_{(\bar{\ell})}=\kappa \theta_{(n)} .
$$

The result is established. As a corollary, the value of the extremality parameter $e$ is the same evaluated for either the constant surface gravity scaling of the null vectors or the corresponding inverse scaling.

In more general situations, deciding on the classification of a horizon will amount to studying the properties of a second order elliptic partial differential operator. In particular, again taking $\left(\ell_{o}, n_{o}\right)$ as reference scalings we have

$$
\delta_{n} \theta_{(\ell)}=-\tilde{d}^{2} \ln f+2 \tilde{\omega}_{o}^{a} \tilde{d}_{a} \ln f+\|\tilde{d} \ln f\|^{2}+\delta_{n_{o}} \theta_{\left(\ell_{o}\right)}
$$

where

$$
\delta_{n_{o}} \theta_{\left(\ell_{o}\right)}=\left\|\tilde{\omega}_{o}\right\|^{2}-\tilde{R} / 2+8 \pi G T_{a b} \ell_{o}^{a} n_{o}^{b},
$$

and we wish to find functions $f$ for which (32) is everywhere negative (or zero or positive).

We will not investigate this equation in detail in this paper but instead content ourselves with proving that, in general, the classification is well defined. That is, given a scaling of the null vectors so that $\delta_{n} \theta_{(\ell)}$ is everywhere negative, it is impossible to rescale the vectors so that the horizon becomes extremal or superextremal. Physically this is equivalent to saying that there cannot be both trapped and untrapped surfaces "just inside" the horizon.

To see this, we reuse Eq. (32) though this time take $\left(\ell_{o}, n_{o}\right)$ as any scaling of the null vectors and $(\ell, n)$ as some rescaling by $f$. Let us assume for a moment that $f$ is analytic. Then if it is not constant it must have maximum and a minimum. At each of these $\tilde{d} \ln f$ vanishes while $-\tilde{d}^{2} \ln f$ is, respectively, greater or less than zero. Thus at $f_{\max }: \delta_{n} \theta_{(\ell)}>\delta_{n_{o}} \theta_{\left(\ell_{o}\right)}$ while at $f_{\min }: \delta_{n} \theta_{(\ell)}<\delta_{n_{o}} \theta_{\left(\ell_{o}\right)}$ and it is clear that if $\delta_{n_{o}} \theta_{\left(\ell_{o}\right)}=0$ then $\delta_{n} \theta_{(\ell)}$ will have a mixed sign. Similarly if $\delta_{n_{o}} \theta_{\left(\ell_{o}\right)}$ is everywhere positive (or negative) then it cannot be rescaled to be everywhere negative (or positive). These results extend to nonanalytic rescalings with the help of the maximum principle (see for example [21]) and so it is clear that rescalings cannot change the classification of a horizon.

Finally, note that we have not eliminated the possibility that some isolated horizons might exist which do not fall into any of the three categories. That is, there may be horizons for which no rescaling will cause $\delta_{n} \theta_{(\ell)}$ to either vanish or be positive/negative everywhere. A source of potential examples are the distorted horizons discussed in [39].

\section{A bound on angular momentum}

With these two notions of extremality established let us now return to the first: is there a maximum angular momentum for rotationally symmetric isolated horizons? In answer to this question we now show that the allowed angular momentum is bound by the intrinsic geometry of the horizon and in particular for a large class of horizons, that bound is exactly the local version of the Kerr bound, Eq. (19). For simplicity, in this section, we restrict our attention to uncharged black holes, i.e. horizons for which the second term in (15) vanishes.

First, applying the Cauchy-Schwarz inequality to the definition of $J[\phi]$, we have

$$
J[\phi]^{2} \leq e \times \frac{1}{16 \pi} \int_{S} d^{2} x \sqrt{\tilde{q}}\|\phi\|^{2},
$$

and so with $e \leq 1$ we immediately have a bound on the angular momentum determined by the intrinsic geometry of the horizon two-surfaces.

To better understand this bound we rewrite it as

$$
J[\phi]^{2} \leq e \gamma R_{H}^{4},
$$

where

$$
\gamma=\frac{\int_{S} d^{2} x \sqrt{\tilde{q}}\|\phi\|^{2}}{16 \pi R_{H}^{4}} .
$$

The properties of $\gamma$ are more easily studied by introducing a canonical coordinate system on $S$. First use the symmetry vector $\phi^{a}$ to generate a foliation of $S$ into circles (plus two poles). Next, choose a point on one of the circles and construct a perpendicular geodesic from that point. This curve runs from pole to pole and also perpendicularly intersects each of the other circles. Then the first coordinate $s$ labels the slices by the proper distance measured along this geodesic from one of the poles. Thus $0 \leq s \leq L$ where $L$ is the distance between the poles. The second coordinate is the usual rotational coordinate $\phi$ defined so that the geodesic is a curve of constant $\phi$ and $\phi^{a} \nabla_{a} \phi=1$. 
For this system the metric takes the form

$$
d S^{2}=d s^{2}+\rho(s)^{2} d \phi^{2},
$$

where $2 \pi \rho(s)$ is the circumference of the circle of coordinate radius $s$ and $\rho(0)=\rho(L)=0$.

Then (36) becomes

$$
\gamma=\frac{\int_{0}^{L} \rho^{3} d s}{2\left(\int_{0}^{L} \rho d s\right)^{2}},
$$

and it is clear that for arbitrary $\rho$ there is no bound on $\gamma-$ given a horizon whose geometry is described by the function $\rho$ and a constant $k$ we can define a new horizon geometry described by $\rho^{\prime}=k \rho$ for which $\gamma^{\prime}=k \gamma$. Thus, $\gamma$ can be made arbitrarily large.

However this class of rescalings is only possible if $d \rho / d s$ is also allowed to become arbitrarily large and, at least in some circumstances, it is reasonable to bound this quantity. For example, if $S$ can be embedded in Euclidean $\mathbb{R}^{3}$ then $d \rho / d s \leq 1$; the maximum rate at which the circumferential radius can increase is $d \rho / d s=1$ (for a flat disc).

Given a bound $|d \rho / d s| \leq m$ for some positive constant $m$, it can be shown that the maximum value of $\gamma$ arises for the curve that increases with slope $m$ as $s$ runs from 0 to $L / 2$ and then decreases with slope $-m$ from $L / 2$ to $L$ (see appendix B). In this case $\gamma=m / 4$ and so (34) becomes

$$
\frac{J[\phi]^{2}}{R_{H}^{4}} \leq \frac{m}{4} .
$$

With $m=1$, the angular momentum is bounded by the standard (Kerr) value [Eq. (19)]. Unfortunately not all surfaces of interest satisfy $|d \rho / d s| \leq 1$. For example it is well known that sufficiently rapidly rotating Kerr horizons cannot be embedded in Euclidean $\mathbb{R}^{3}$. This is precisely because in this situation $|d \rho / d s|>1$ near the poles. For extremal Kerr it achieves a maximum value of $3 \sqrt{3} / 4 \approx$ 1.299 and $\gamma=\pi / 4-1 / 2 \approx 0.285$. Therefore, the local extremality condition (21) cannot be used to infer $J \leq$ $R_{H}^{2} / 2$ even though this condition still holds. Interestingly, as shown in Appendix A, for the Kerr-AdS black holes $J \leq$ $R_{H}^{2} / 2$ may be violated by an arbitrary amount. In such cases $e \leq 1$ and the new bound (39) still holds although with $m=|d \rho / d s|_{\max }>1$.

In summary the allowed angular momentum for a rotationally symmetric isolated horizon is bound by the intrinsic geometry of the surface. For surfaces that can be embedded in Euclidean $\mathbb{R}^{3}$ this is exactly the usual Kerr bound. However for more exotic horizon cross sections the intrinsic geometry can be similarly exotic and so the numerical factor $m$ in (39) can become arbitrarily large.

\section{DYNAMICAL HORIZONS}

Let us now turn our attention to local, interacting horizons. There are several formulations describing these hori- zons, but here we choose to use the dynamical horizon framework of Ashtekar and Krishnan [17,18]. The results are equally applicable, with minor modifications, to the other formulations, including Hayward's trapping horizons [9-11].

We begin by recalling a few basic properties of dynamical horizons. A dynamical horizon $H$ is a spacelike threesurface, uniquely [22] foliated by two-surfaces which are marginally trapped, namely $\theta_{(\ell)}=0$ and $\theta_{(n)}<0$. Given a foliation label $v$ we can write the evolution vector field $\mathcal{V}^{a}$, defined so that it is normal to the two-surfaces and $\mathcal{L}_{\mathcal{V}} v=1$, as

$$
\mathcal{V}^{a}=A \ell^{a}-B n^{a}
$$

for some functions $A$ and $B$. Since $\mathcal{V}^{a}$ is tangent to the spacelike $H$, neither $A$ nor $B$ can vanish anywhere. Further if we choose the orientation of the labelling so that $A>0$ it follows that $B>0$ as well. From this and the assumption that $\theta_{(n)}<0$, it immediately follows that, like event horizons, dynamical horizons always expand in area:

$$
\mathcal{L}_{\mathcal{V}} \sqrt{\tilde{q}}=-\sqrt{\tilde{q}} B \theta_{(n)} .
$$

We now consider the possible characterizations of extremality. The ambiguities in defining a mass or energy are even greater than for isolated horizons so we do not pursue that characterization. There are also difficulties in using surface gravity. While this can be defined analogously to the surface gravity on an isolated horizon:

$$
\kappa=-\mathcal{V}^{a} n_{b} \nabla_{a} \ell^{b},
$$

it is generally not constant on a dynamical horizon. This is to be expected as, taking the analogy between black holes and thermodynamics, it is equivalent to the statement that the temperature will not normally be constant for a system away from equilibrium. Thus, for dynamical horizons we have little control over the value of the surface gravity, and hence cannot use it in constructing an extremality condition.

A partial exception to this statement occurs if the horizon is slowly evolving as defined in [19-21]. In this case it is in quasiequilibrium and "almost" isolated and one can show that the surface gravity almost constant. It changes slowly both across the two-surface cross sections and in evolving up the horizon. In this case it would be feasible to state that the horizon is nonextremal, at least to the order for which $\kappa$ is constant.

More generally we are left to consider the implication of the existence of fully trapped surfaces just inside the horizon. Here, we restrict attention to "generic dynamical horizons" in the sense of [22], in order that we are dealing only with black hole horizons and not cosmological horizons or horizons arising in other spacetimes, such as those in [46]. The genericity condition requires that $\delta_{\ell} \theta_{(\ell)}$ does not vanish at any point on the horizon. As has been shown previously, for example see Refs. $[8,18]$, this guarantees 
that there will be trapped surfaces inside the horizon. Here we briefly repeat the argument.

First, scale the null vectors so that

$$
\stackrel{\ell}{\leftarrow} d v,
$$

or equivalently

$$
\ell^{a}=f(v)\left(\hat{r}^{a}+\hat{\tau}^{a}\right),
$$

where $f(v)$ is a positive function, $\hat{\tau}^{a}$ is the future-pointing timelike normal to the horizon and $\hat{r}^{a}$ is the in-horizon spacelike normal to the slices that points in the direction of increasing area. For such a scaling, $B$ in Eq. (40) is constant on cross sections of the horizon whence Eq. (24) simplifies to

$$
\mathcal{L}_{\mathcal{V}} \theta_{(\ell)}=A \delta_{\ell} \theta_{(\ell)}-B \delta_{n} \theta_{(\ell)} .
$$

Thus, with $\theta_{(\ell)}$ zero everywhere on $H$,

$$
\delta_{n} \theta_{(\ell)}=\left(\frac{A}{B}\right) \delta_{\ell} \theta_{(\ell)} .
$$

Finally if the null energy condition holds and we assume that $\delta_{\ell} \theta_{(\ell)}$ nowhere vanishes, then by Eq. (25) we have

$$
\delta_{n} \theta_{(\ell)}<0 .
$$

Since trapped surfaces must exist inside a dynamical horizon, we can immediately apply the results of sections III C and III D to them. In particular, it follows that the extremality parameter $e$ introduced in Eq. (21) cannot exceed unity. Further, by Eq. (47) it must be strictly less than one-dynamical horizons must be subextremal. Thus, for example, in Einstein-Maxwell theory the angular momentum one-form and electric and magnetic fluxes are bound on a dynamical horizon by

$$
\frac{1}{4 \pi} \int_{S_{v}} d^{2} x \sqrt{\tilde{q}}\left(\|\tilde{\omega}\|^{2}+G\left(E_{\perp}^{2}+B_{\perp}^{2}\right)\right)<1,
$$

and for an axisymmetric horizon whose cross sections can be embedded in Euclidean $\mathbb{R}^{3}$ :

$$
J[\phi]^{2}<R_{H}^{4} / 4 .
$$

While dynamical horizons cannot violate the trapped surface extremality condition, they can transform into a new type of structure that does not contain trapped surfaces inside. In [47], it is demonstrated that marginally trapped tubes (foliated three-surfaces which satisfy $\theta_{(\ell)}=0$ and $\left.\theta_{(n)}<0\right)$ in Tolman-Bondi spacetimes may transform from being spacelike (and so dynamical horizons with $\delta_{n} \theta_{(\ell)}<$ 0 ) to timelike (with $\delta_{n} \theta_{(\ell)}>0$ ). The change in behavior occurs when the dust density $\rho$ becomes greater than $1 / a$ where $a$ is the area of the horizon cross sections. Intuitively one can think of this as occurring when the matter density becomes high enough to form a new horizon outside the old and then the timelike section of the horizon is charac- teristic of a horizon "jump." Such a behavior is shown in Fig. 2 (which is adapted from [47]).

Similarly, Schnetter, Krishnan, and Florian [30] have studied various numerical simulations including the collision of spinning black holes. They find that before the holes collide, an outer spacelike horizon forms. At the same time, an inner horizon forms which is part spacelike, part timelike. Although they were unable to follow the evolution far enough, they conjecture that the horizons will form a continuous three-surface, only some fraction of which is spacelike. The results presented here suggest a criterion for determining when these jumps are about to occur - the transition between the spacelike and timelike sections of the horizon will occur as $e \rightarrow 1$. Note however that in general this transition may be complicated and include horizon cross sections whose evolution may be spacelike in some areas and null or timelike in others. For a detailed understanding of the transition one would need to track $\delta_{n} \theta_{(\ell)}$ and/or the signature of the evolution vector point-by-point. However while the evolution is still purely spacelike, $e$ should provide a good estimate of the proximity to extremality.

With a view towards tracking either $e$ or $\delta_{n} \theta_{(\ell)}$ in a simulation, let us reformulate the expressions above in terms of spacelike/timelike unit normals. First, in terms of the unit tangent $\left(\hat{r}^{a}\right)$ and normal $\left(\hat{\tau}^{a}\right)$ vectors to the horizon, the angular momentum one-form and stressenergy component can be rewritten as

$$
\tilde{\omega}_{a}=\tilde{q}_{a}^{b} \hat{r}^{c} K_{b c}^{(\hat{\tau})} \quad \text { and } \quad T_{a b} \ell^{a} n^{b}=T_{a b}\left(\hat{\tau}^{a} \hat{\tau}^{b}-\hat{r}^{a} \hat{r}^{b}\right)
$$

for the preferred scaling (44) where $K_{b c}^{(\hat{\tau})}$ is the extrinsic curvature of the horizon relative to $\hat{\tau}$.

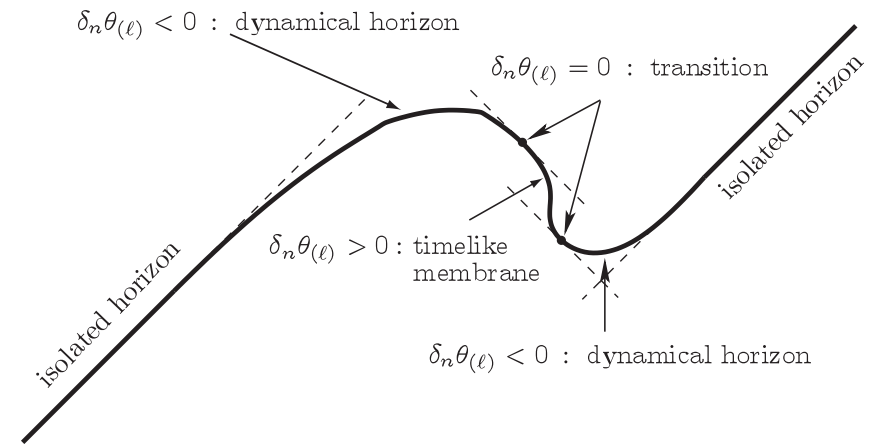

FIG. 2. A schematic of a horizon "jump." Matter falls into an isolated horizon causing it to expand as a dynamical horizon. However at a certain point the density of the matter is such that a new horizon forms outside the old resulting in a jump which geometrically corresponds to a timelike membrane (a timelike three-surface with $\left.\theta_{(\ell)}=0, \theta_{(n)}<0\right)$ connecting two dynamical horizons. In this figure $45^{\circ}$ lines are null, time increases in the vertical direction, and surface area increases to the right. 
Alternatively we can consider an apparent horizon, with unit normal $\hat{s}$, in a three-slice $\Sigma_{t}$, with unit timelike normal $\hat{u}$. The horizon evolution vector field $\mathcal{V}$ can be expressed as

$$
\mathcal{V}^{a}=N\left(\hat{u}^{a}+v_{\perp} \hat{s}^{a}\right)
$$

where $N$ is the lapse and $v_{\perp}$ is the velocity of the horizon relative to the foliation. Then, following [48], we can write

$$
\delta_{n} \theta_{(\ell)}=-\frac{1}{2}\left(\frac{v_{\perp}+1}{v_{\perp}-1}\right)\left(\left\|\sigma_{(\underline{\ell})}\right\|^{2}+8 \pi G T_{a b} \underline{\ell}^{a} \underline{\ell}^{b}\right)
$$

where $\underline{\ell}^{a}=\hat{u}^{a}+\hat{s}^{a}$ and so $\sigma_{(\underline{\ell}) a b}=\tilde{q}_{a}^{c} \tilde{q}_{b}^{d}\left(K_{c d}+D_{c} \hat{s}_{d}\right)$. We obtain a similar expression for the extremality parameter $e$ as:

$$
\begin{aligned}
e= & 1+\frac{1}{4 \pi G} \int_{S_{t}} d^{2} x \sqrt{\tilde{q}} \delta_{n} \theta_{(\ell)} \\
= & 1-\frac{1}{8 \pi G} \int_{S_{t}}-d^{2} x \sqrt{\tilde{q}}\left(\frac{v_{\perp}+1}{v_{\perp}-1}\right) \\
& \times\left(\left\|\sigma_{(\underline{\ell})}\right\|^{2}+8 \pi G T_{a b} \underline{\ell}^{a} \underline{\ell}^{b}\right) .
\end{aligned}
$$

See Ref. [48] for further details of these calculations.

Thus, thanks to the preferred scaling of the null normals we have an unambiguous definition of $e$ on each slice of any dynamical horizon. Things are, however, slightly more complicated in more general situations. First, keep in mind that the scalings (44) are defined by the timelike normal to $H$ and the spacelike normal to $S_{v}$ in $H$. Thus one cannot use this form of the definition if $H$ becomes null (either as an isolated horizon or while transitioning to become a timelike membrane). In such cases one must return to the original definition Eq. (21).

There is also a second situation where it is not feasible to use Eq. (53) to calculate $e$. An apparent horizon $S$ in a set of initial data can evolve into many different dynamical horizons depending on how the data itself is evolved - that is apparent horizons are foliation dependent. From the point of view of Eq. (53) the various potential horizons will generate different scalings of the null normals to $S$ and so different values of $e$. This ambiguity can be more easily understood by switching back to the original definition of the extremality parameter given in Eq. (21). Then, if $\ell^{\prime}=$ $f \ell$ and $n^{\prime}=n / f$ the ambiguity in $e$ under rescalings of the null vectors is given by

$$
e^{\prime}=e+\frac{1}{4 \pi} \int_{S} d^{2} x \sqrt{\tilde{q}}\left(2 \tilde{\omega}^{a} \tilde{d}_{a} \ln f+\|d \ln f\|^{2}\right)
$$

while

$$
\delta_{n^{\prime}} \theta_{\left(\ell^{\prime}\right)}=\delta_{n} \theta_{(\ell)}-\tilde{d}^{2} \ln f+\|d \ln f\|^{2}+2 \tilde{\omega}^{a} \tilde{d}_{a} \ln f .
$$

That said, it should be kept in mind that by the trapped surface classification presented in Sec. III C, $S$ is defined as subextremal, extremal, or superextremal based on possible rather than any particular scalings of the null vectors. Though $e$ may vary for various choices of scalings, the ultimate classification of $S$ is invariant. For dynamical horizons a suitable scaling is defined by the normals to $H$, but if one only has a single surface, then one must go back to an analysis of the elliptic operator defined by Eq. (55).

Interestingly, in the context of trapping horizons, Hayward [8] has introduced an alternative expression for surface gravity which is proportional to $\sqrt{-\delta_{n} \theta_{(\ell)}}$. Such a definition explicitly ties together the nonvanishing of surface gravity with the existence of trapped surfaces inside the horizon, i.e. the second and third characterizations of extremality necessarily coincide. Furthermore, making use of Eq. (20) he has obtained a zeroth law for trapping horizons which has many similarities with the extremality condition introduced in this paper.

\section{SUMMARY}

In this paper, we have considered three characterizations of extremality. The first is the standard Kerr bound on angular momentum relative to mass. We have argued that in general it is not well-posed due to the difficulties in defining mass and angular momentum in general relativity. Even when the Kerr bound is reformulated in terms of horizon area and angular momentum, it can only be meaningfully evaluated on axisymmetric horizons. Furthermore, while we have not provided an explicit violation of this bound in asymptotically flat spacetimes, we have argued that it is likely that it can be violated. In particular KerrAdS solutions can violate the bound by an arbitrary amount. These results do not violate the recent theorems of Dain which only apply to asymptotically flat vacuum spacetimes.

A more satisfactory characterization of extremality for isolated horizons arises from the surface gravity. For isolated horizons, a subextremal horizon will have positive surface gravity, while the surface gravity for an extremal horizon vanishes. Furthermore, non-negativity of surface gravity leads to a bound on the integrated square of the angular momentum density and the matter stress-energy at the horizon.

Alternatively, we can characterize nonextremality as the requirement that there should be fully trapped surfaces just inside a black hole horizon. This notion is then applicable to both isolated and dynamical horizons. In addition, this condition again leads to a bound on the integrated square of the angular momentum density and the matter stressenergy at the horizon. The surface gravity and trapped surface characterizations of extremality for isolated horizons are very closely related, and indeed in axisymmetry are entirely equivalent.

The local extremality condition is also sufficient to place a restriction on the maximum allowed angular momentum relative to the intrinsic geometry of the horizon. For hori- 
zons whose cross sections can be embedded in Euclidean $\mathbb{R}^{3}$ this is sufficient to imply the standard Kerr bound $4 J^{2}<$ $R_{H}^{4}$, however for more exotic intrinsic geometries we can only show that $4 J^{2}<m R_{H}^{4}$ for some constant $m$ which may be made arbitrarily large in, for example, Kerr-AdS.

Thus, the notion of extremality extends beyond the Kerr solutions though in a nontrivial way. The spirit of the bounds remains. The angular momentum of the horizon is bounded relative to the intrinsic geometry of the horizon. In general, when no axis of rotation exists, it is the square of the angular momentum density which is bounded. Equivalently, one can think of this as a bound on the sum of the multipole moments of the angular momentum rather than the dipole itself. The extremality quantity $e$, which we have introduced should be calculable on apparent horizons occurring in numerical relativity simulations and would provide an interesting characterization of how close to extremality a black hole is immediately following a merger.

\section{ACKNOWLEDGMENTS}

We would like to thank Peter Booth, Patrick Brady, Jolien Creighton, Herb Gaskill and Badri Krishnan for helpful discussions. Ivan Booth was supported by the Natural Sciences and Engineering Research Council of Canada. Stephen Fairhurst was supported by NSF Grant No. PHY-0200852 and the Royal Society.

\section{APPENDIX A: KERR ANTI-DESITTER BLACK HOLES}

The Kerr-(anti)deSitter family of solutions are described by the metric:

$$
\begin{aligned}
d s^{2}= & -\frac{\Delta}{\rho^{2}}\left(d t-\frac{a}{\Xi} \sin ^{2} \theta d \phi\right)^{2}+\frac{\rho^{2}}{\Delta} d r^{2}+\frac{\rho^{2}}{\Delta_{\theta}} d \theta^{2} \\
& +\frac{\Delta_{\theta} \sin ^{2} \theta}{\rho^{2}}\left(a d t-\frac{r^{2}+a^{2}}{\Xi} d \phi\right)^{2}
\end{aligned}
$$

where

$$
\begin{aligned}
& \Delta=-\frac{\Lambda}{3} r^{4}+\left(1-\frac{\Lambda}{3} a^{2}\right) r^{2}-2 M r+a^{2}, \\
& \Delta_{\theta}=1+\frac{\Lambda}{3} a^{2} \cos \theta^{2}, \\
& \Xi=1+\frac{\Lambda}{3} a^{2} \quad \text { and } \quad \rho^{2}=r^{2}+a^{2} \cos ^{2} \theta .
\end{aligned}
$$

$\Lambda$ is the cosmological constant (and so is positive for deSitter and negative for anti-de Sitter), $M$ is the mass parameter, and $a$ is the rotation parameter.

We are interested in the black hole sector of the solution space. The various horizons occur at the roots of $\Delta=0$. For $\Lambda>0, \Delta$ has four roots in the black hole sector. In increasing order they are a (negative) unphysical solution, inner black hole horizon, outer black hole horizon, and the cosmological horizon. For $\Lambda<0$ there are just two roots: the inner and outer black hole horizons. Our interest is in the outer black hole horizon which we label $r_{+}$. The coordinate representation (A1) of the metric diverges at $r_{+}$but for our purposes we can work around this by considering appropriate limiting cases which are welldefined. Then, one can show (see for example [49]) that the areal radius and angular momentum of the horizon are, respectively,

$$
R_{H}^{2}=\frac{r_{+}^{2}+a^{2}}{\Xi} \quad \text { and } \quad J[\phi]=\frac{M a}{\Xi^{2}} .
$$

Here, for definiteness, we focus on the extremal horizons of this family where the inner and outer horizons coincide and so $\Delta$ has a degenerate root (the second and third roots are degenerate for $\Lambda>0$ ). Such cases are most easily identified by examining where the discriminant of $\Delta$ vanishes (the expression is a quintic in $a^{2}$ and quartic in $\Lambda$ but may be dealt with easily enough with the help of a computer algebra system).

For a given value of the mass $M$, there is a finite range of $\Lambda$ for which extremal solutions exist. The lower bound is $\Lambda_{\text {min }} \approx-7.1 / M^{2}$ where $\Xi=0$ (this is a lower bound as for $\Xi<0$ the signature of the $\theta$ coordinate changes and becomes timelike close to 0 and $\pi$ ). The maximum value $\Lambda_{\max } \approx 0.18 / M^{2}$ occurs when the inner and outer black hole horizons and the cosmological horizon all coincide in a triply degenerate root.

Given the range of values of $\Lambda$ which permit an extremal horizon, we can plot $J / R_{H}^{2}$ and see whether the extremality bound (19) is violated. This is shown in Fig. 3 and it is clear that the bound $J \leq R_{H}^{2} / 2$ is violated for all extremal KerrAdS solutions. To understand this in light of the discussion in Section III D, first note that in the presence of a cosmological constant Eq. (21) becomes:

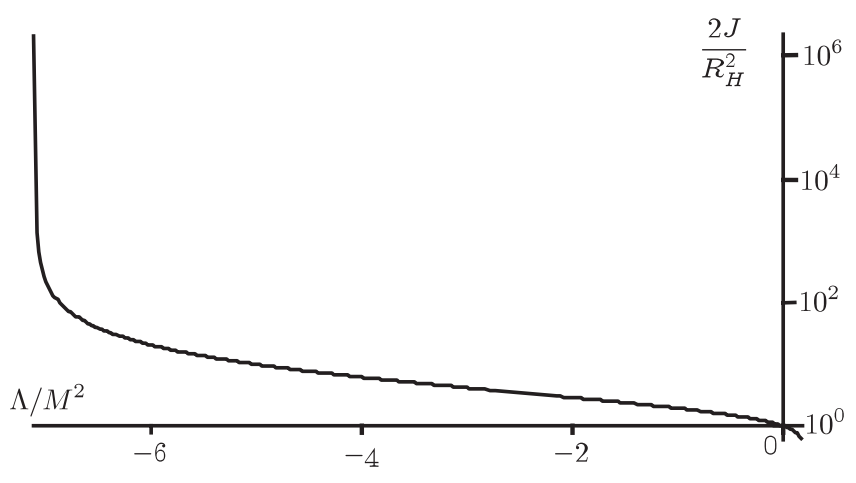

FIG. 3. $2 J / R_{H}^{2}$ versus $\Lambda$ for the extremal Kerr-(A)dS family of black holes. For $\Lambda=0,2 J / R_{H}^{2}=1$, as expected for the extremal, asymptotically flat Kerr solution. For $\Lambda>0,2 J / R_{H}^{2}<1$ while for $\Lambda<0, J / R_{H}^{2}>1 / 2$ and it diverges as $\Lambda$ approaches its minimum allowed value. Therefore, for extremal Kerr antide Sitter black holes, the local reformulation of the Kerr bound (19) is violated. 


$$
e:=\int_{S_{v}} d^{2} x \sqrt{\tilde{q}}\left(2 G T_{a b} \ell^{a} n^{b}+\frac{1}{4 \pi}\|\tilde{\omega}\|^{2}-\Lambda\right) \leq 1,
$$

Thus for $\Lambda<0$, the contribution from the cosmological constant is positive and so we still have $J^{2} / R_{H}^{4} \leq m / 4$. In this case however $m$ becomes arbitrarily large as we approach $\Xi=0$. Specifically, the induced metric on a cross section of the horizon is

$$
d S^{2}=\frac{\rho^{2}}{\Delta_{\theta}} d \theta^{2}+\frac{\Delta_{\theta}\left(r^{2}+a^{2}\right)^{2} \sin ^{2} \theta}{\rho^{2} \Xi^{2}} d \phi^{2} .
$$

Then the circumferential radius is

$$
R=\frac{\sqrt{\Delta_{\theta}}\left(r^{2}+a^{2}\right) \sin \theta}{\rho \Xi}
$$

and

$$
m=\max \left(\frac{\sqrt{\Delta_{\theta}}}{\rho} \frac{d R}{d \theta}\right)
$$

It is easy to see that this quantity diverges as $\Xi \rightarrow 0$.

For simplicity we only considered extremal horizons here, but it is clear (by continuity) that these violations of the bound will also extend into parts of the nonextremal sector.

Finally, it is perhaps interesting to note that on inserting a $\Lambda>0$ into Eq. (A4), we see that the upper bound on the integral of $\|\tilde{\omega}\|^{2}$ increases with increasing $\Lambda$. However, at least for Kerr-dS this does not provide enough freedom to violate $J^{2} \leq R_{H}^{4}$ as there is a concomitant tightening of $m$.

\section{APPENDIX B: SURFACE OF MAXIMUM $\gamma$}

Let $R$ be the set of continuous functions $\rho(s):[0, L] \rightarrow$ $\mathbb{R}$ that satisfy

(i) $\rho(s) \geq 0$

(ii) $\rho(0)=\rho(L)=0$

(iii) $d \rho / d s(0)>0$ and $d \rho / d s(L)<0$ and

(iv) $|d \rho / d s| \leq m$ for some $m>0$.

Thinking back to the two-surfaces defined by these $\rho$, the first condition guarantees a non-negative "radius," the second and third require that the surfaces close exactly at 0 and $L$, and the fourth is the assumed bound on the maximum rate of change of the radius relative to the arclength.

Further define

$$
\gamma=\frac{\int_{0}^{L} \rho^{3} d s}{2\left(\int_{0}^{L} \rho d s\right)^{2}} .
$$

Then in this appendix we show that of all $\rho \in R$, the triangular function

$$
\rho_{\triangle}(s)=\left\{\begin{array}{ll}
m s & 0 \leq s \leq L / 2 \\
m(L-s) & L / 2 \leq L
\end{array},\right.
$$

shown in Fig. 4 maximizes $\gamma$.

This is slightly more complicated than a basic variational problem. As noted in the text, if one generalizes to the set of all non-negative functions then $\gamma$ is unbounded. Thus, our goal is to show that $\gamma$ is globally maximized over $R$ by the "boundary" curve $\rho_{\triangle}$.

To prove this we first show that $\rho_{\triangle}$ gives a local maximum. To this end, we calculate the first variation of $\gamma$ in $\rho$ as

$$
\delta \gamma=\frac{\left(3 \int \rho^{2} \delta \rho d s\right)\left(\int \rho d s\right)-2\left(\int \delta \rho d s\right)}{2\left(\int \rho d s\right)^{3}},
$$

where all integrals are from 0 to $L$. For variations around $\rho_{\triangle}$ this becomes

$$
\delta \gamma_{\triangle}=\frac{8}{L^{4}} \int_{0}^{L / 2}\left(12 s^{2}-L^{2}\right)(\delta \rho(s)+\delta \rho(L-s)) d s .
$$

Now by the restriction on the maximum slope, all allowed $\delta \rho \leq 0$ and further $\delta \rho$ is nonincreasing from 0 to $L / 2$. Thus taking $s_{o}=L /(2 \sqrt{3})$ (the zero of $12 s^{2}-L^{2}$ ) as a dividing point, we have $|\delta \rho(s)| \leq \delta \rho\left(s_{o}\right)$ for $s \in\left[0, s_{o}\right]$ and $|\delta \rho(s)| \geq \delta \rho\left(s_{o}\right)$ for $s \in\left[s_{o}, L / 2\right]$. Similar results apply for $\delta \rho(L-s)$ which is also nonincreasing on this interval. Then, keeping in mind that $\delta \rho$ must be zero at least somewhere we find $\delta \gamma_{\triangle}<0$. That is, all allowed variations decrease the value of $\gamma$ and so $\rho_{\triangle}$ provides at least a local maximum for our problem.

We complete the proof by showing for any other $\rho \in R$ we can find a $\gamma$-increasing variation $\delta \rho$. It will be sufficient to restrict our attention to the subset of variations for which $\int-\delta \rho d s=0$. For such variations (B2) simplifies and we find

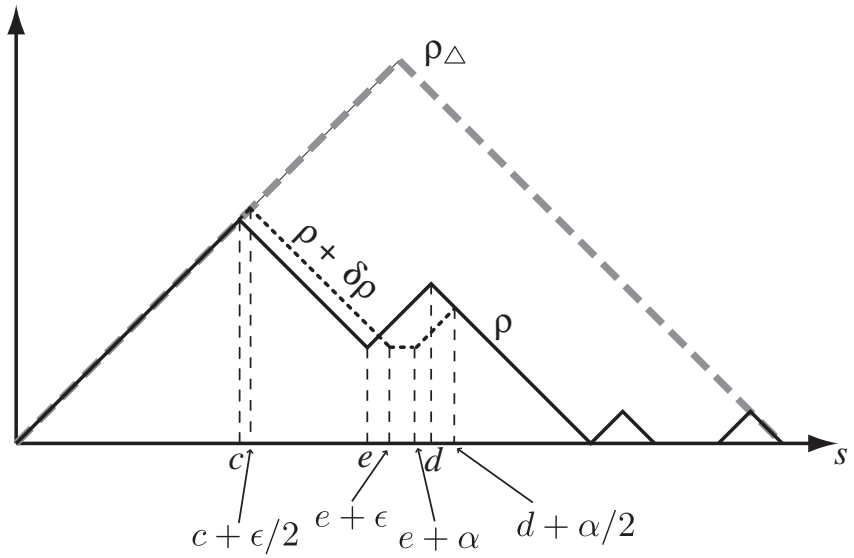

FIG. 4. Several $\rho$ that appear in the text. $\rho_{\triangle}$ is the maximizing curve, $\rho$ is a typical "saw-toothed" curve, and $\rho+\delta \rho$ (which appears as a dotted line where it does not coincide with $\rho$ ) is a variation of that curve which increases the value of $\gamma$. 


$$
\delta \gamma>0 \Leftrightarrow \int-\rho^{2} \delta \rho d s>0 .
$$

Intuitively these inequalities can be satisfied by constructing variations which increase $\rho$ where it is larger and balancing this off by decreasing it where it is smaller.

First consider the case where there is an interval $[a, b]$ over which $\rho$ is monotonically increasing but $d \rho / d s<m$ and construct a variation

$$
\delta \rho= \begin{cases}0 & 0 \leq s<a \\ -\epsilon \sin \left(2 \pi\left(\frac{s-a}{b-a}\right)\right) & a \leq s \leq b, \\ 0 & b<s \leq b\end{cases}
$$

where $\epsilon$ is arbitrarily small; in particular, it is sufficiently small to ensure that $\rho+\delta \rho>0$ and $|d(\rho+\delta \rho) / d s|<m$. By the monotonicity $\rho(s)<\rho((a+b) / 2)$ for $s \in[a,(a+$ $b) / 2)$ and $\rho(s)>\rho((a+b) / 2)$ for $s \in((a+b) / 2, b]$, so by (B4) it is straightforward to see that $\delta \gamma>0$. Thus any $\rho$ that contains an increasing region over which $d \rho / d s<m$, cannot maximize $\gamma$. A nearly identical argument shows that an $\rho$ with a decreasing region over which $-m<$ $d \rho / d s$ cannot provide a maximum.

In fact the same variation (B5) can also be used to eliminate all $\rho$ which contain a constant section $[a, b]$ over which $\rho=\rho_{o}>0$. In that case $\delta \gamma$ vanishes but a straightforward calculation of the second variation shows that this is because such a $\rho$ is a local minimum with respect to these variations.

Thus a $\rho$ which maximizes $\gamma$ must have slope \pm 1 everywhere-that is either $\rho_{\triangle}$ or a (possibly broken) "saw-toothed" curve such as that shown in Fig. 4. There are several special cases to consider here but while details differ, the basic variation is the same: we increase a higher peak while decreasing a lower one and so increase $\gamma$. In the interests of saving space we consider only the case of two immediately adjoining peaks as shown in the figure.

Then, with the higher peak at $c$, lower at $d$ and the valley in between at $e$ we consider variations of the following type:

$$
\delta \rho=\left\{\begin{array}{ll}
2 m(s-c) & c \leq s \leq c+\epsilon / 2 \\
m \epsilon & c+\epsilon / 2 \leq e \\
m \epsilon-2 m(s-e) & e \leq s \leq e+\epsilon \\
-m(s-e) & e+\epsilon \leq s \leq e+\alpha \\
-m \alpha & e+\alpha \leq s \leq d \\
-m \alpha+2 m(s-d) & d \leq s \leq d+\alpha / 2
\end{array} .\right.
$$

$\epsilon$ is the usual small parameter and $\alpha$ is chosen so that $\int \delta \rho d s=0$. To first order (which is all that is needed for a variational calculation) it is

$$
\alpha \approx\left(\frac{e-c}{d-e}\right) \epsilon .
$$

Then a direct calculation with (B4) shows that if the first peak is higher than the second, $\delta \gamma>0$. If they are equal then $\delta \gamma=0$ but going to the second order variation, it can be seen that this is because it is a local minimum under such variations. Similarly ponderous calculations can be performed to show that no other "saw-toothed" $\rho$ is a maximum.

Thus in summary we have shown that $\rho_{\triangle}$ is a local maximum for curves in $R$ while there exist variations of all other curves that increase $\gamma$. Thus, $\rho_{\triangle}$ is the global maximum as claimed.
[1] S. Dain, arXiv:gr-qc/0606105.

[2] S. Dain, Phys. Rev. Lett. 96, 101101 (2006).

[3] S. Dain, Classical Quantum Gravity 23, 6845 (2006).

[4] M. Ansorg and D. Petroff, Phys. Rev. D 72, 024019 (2005).

[5] M. Ansorg and D. Petroff, Classical Quantum Gravity 23, L81 (2006).

[6] D. Petroff and M. Ansorg, arXiv:gr-qc/0511102.

[7] L. B. Szabados, Living Rev. Relativity 7, 4 (2004).

[8] S. A. Hayward, Phys. Rev. D 49, 6467 (1994).

[9] S. A. Hayward, Phys. Rev. D 70, 104027 (2004).

[10] S. A. Hayward, arXiv:gr-qc/0607081.

[11] S. A. Hayward, Phys. Rev. Lett. 93, 251101 (2004).

[12] A. Ashtekar, C. Beetle, and S. Fairhurst, Classical Quantum Gravity 16, L1 (1999).

[13] A. Ashtekar, C. Beetle, and S. Fairhurst, Classical Quantum Gravity 17, 253 (2000).

[14] A. Ashtekar, S. Fairhurst, and B. Krishnan, Phys. Rev. D 62, 104025 (2000)
[15] A. Ashtekar et al., Phys. Rev. Lett. 85, 3564 (2000).

[16] A. Ashtekar, C. Beetle, and J. Lewandowski, Phys. Rev. D 64, 044016 (2001).

[17] A. Ashtekar and B. Krishnan, Phys. Rev. Lett. 89, 261101 (2002).

[18] A. Ashtekar and B. Krishnan, Phys. Rev. D 68, 104030 (2003).

[19] I. Booth and S. Fairhurst, Phys. Rev. Lett. 92, 011102 (2004).

[20] W. Kavanagh and I. Booth, Phys. Rev. D 74, 044027 (2006).

[21] I. Booth and S. Fairhurst, Phys. Rev. D 75, 084019 (2007).

[22] A. Ashtekar and G. J. Galloway, Adv. Theor. Math. Phys. 9, 1 (2005).

[23] J. Lewandowski and T. Pawlowski, Classical Quantum Gravity 20, 587 (2003).

[24] R. Penrose, Phys. Rev. Lett. 14, 57 (1965).

[25] W. Israel, Phys. Rev. Lett. 57, 397 (1986).

[26] A. Ashtekar, C. Beetle, and J. Lewandowski, Classical 
Quantum Gravity 19, 1195 (2002).

[27] I. Booth and S. Fairhurst, Classical Quantum Gravity 22, 4515 (2005).

[28] J. D. Brown J. York, and W. James, Phys. Rev. D 47, 1407 (1993).

[29] O. Dreyer, B. Krishnan, D. Shoemaker, and E. Schnetter, Phys. Rev. D 67, 024018 (2003).

[30] E. Schnetter, B. Krishnan, and F. Beyer, Phys. Rev. D 74, 024028 (2006).

[31] S. A. Hayward, Phys. Rev. D 74, 104013 (2006).

[32] G. B. Cook and B.F. Whiting, Phys. Rev. D 76, 041501 (2007).

[33] M. Korzynski, Classical Quantum Gravity 24, 5935 (2007).

[34] D. Christodoulou, Phys. Rev. Lett. 25, 1596 (1970).

[35] R. C. Myers and M. J. Perry, Ann. Phys. (N.Y.) 172, 304 (1986).

[36] R. Emparan and R. C. Myers, J. High Energy Phys. 09 (2003) 025.

[37] M. Ansorg and H. Pfister, Classical Quantum Gravity 25,
035009 (2008).

[38] T. Pawlowski, J. Lewandowski, and J. Jezierski, Classical Quantum Gravity 21, 1237 (2004).

[39] S. Fairhurst and B. Krishnan, Int. J. Mod. Phys. D 10, 691 (2001).

[40] A. Ashtekar, J. Engle, T. Pawlowski, and C. Van Den Broeck, Classical Quantum Gravity 21, 2549 (2004).

[41] R.P. A. C. Newman, Classical Quantum Gravity 4, 277 (1987).

[42] E. Gourgoulhon, Phys. Rev. D 72, 104007 (2005).

[43] E. Gourgoulhon and J.L. Jaramillo, Phys. Rev. D 74, 087502 (2006).

[44] M. Korzynski, Phys. Rev. D 74, 104029 (2006).

[45] D. M. Eardley, Phys. Rev. D 57, 2299 (1998).

[46] J. M. M. Senovilla, J. High Energy Phys. 11 (2003) 046.

[47] I. Booth, L. Brits, J. A. Gonzalez, and C. Van Den Broeck, Classical Quantum Gravity 23, 413 (2006).

[48] I. Booth, arXiv:0709.0934.

[49] M. H. Dehghani and R. B. Mann, Phys. Rev. D 64, 044003 (2001). 